\title{
Relation-Oriented Resource Allocation for Multi-Accelerator Systems
}

\author{
Teng Yu*, Bo Feng ${ }^{\dagger}$, Mark Stillwell ${ }^{\S}$, Jose Gabriel F Coutinho*, Wenlai Zhao ${ }^{\dagger}$, Shuang Liang ${ }^{\dagger}$, \\ Wayne Luk*, Alexander L. Wolf*, Yuchun $\mathrm{Ma}^{\dagger}$ \\ ${ }^{*}$ Imperial College London, UK, ${ }^{\dagger}$ Tsinghua University, China, ${ }^{\S}$ Cisco Meraki, UK \\ Email: t.yu15@imperial.ac.uk
}

\begin{abstract}
This paper presents a novel approach for allocating resources in systems with multiple accelerators. It has three main contributions. First, a new model based on Birkhoff's representation theory in capturing the ordering properties of resource allocation requests (RArs). Second, an effective technique for resource allocation based on this model, targeting systems with multiple accelerators. Third, the evaluation of the proposed approach for Maxeler MPC-X multi-accelerator systems, demonstrating time-efficiency and $30 \%-50 \%$ failure-rate decrease (FRD) on random input dataset.
\end{abstract}

\section{INTRODUCTION}

In this paper, we provide a generalised procedure for developing allocators that can handle resources with arbitrary capacity and allocation request representations for multi-accelerator systems. Current resource allocators, such as OpenStack [6] for VMs, Maxeler Orchestrator [5] for FPGA-based Data Flow Engines (DFEs), and XtreemFS [8] for distributed file systems require specific interpretation of the representation of RArs and capacity of resources. In particular, RArs associated to each type must be interpreted so that they can be ranked, which has been shown to optimise the allocation process [7].

To generalise the allocation process, we come up with a novel approach for ranking arbitrary heterogeneous RArs for multi-accelerator systems: the ranking values are universally consistent to satisfy the uniformity on heterogeneous resources and they are easy-computable, within polynomial-time, to satisfy time efficiency constraints. To obtain uniform and efficient ranking values, we consider a partially ordered model and apply Birkhoff's representation theory [1] in capturing the ordering properties of arbitrary RArs. As for the allocation procedure, the allocator uses the ranking values of arbitrary heterogeneous RArs to guide the allocation process to reduce failure rate (FR) and preserve time-efficiency on multi-accelerator systems.

The proposed model with the corresponding resource allocator has been implemented and evaluated on a case study for the Maxeler MPC-X multi-accelerator system [5]. Our experimental results have demonstrated time-efficiency and a significant decrease in FR on random input data compared with the current commonly used First In First Out (FIFO) resource allocation/scheduling method.

\section{Modelling Multi-Accelerator Systems}

An abstract taxonomy of modern multi-accelerator systems with different types of accelerators and different topologies

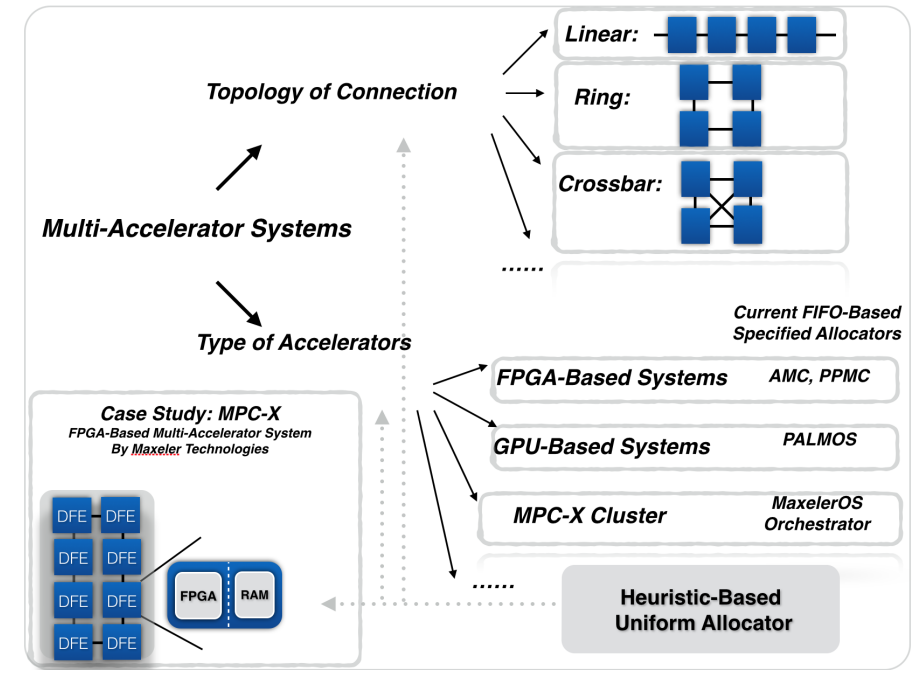

Figure 1: Multi-Accelerator Systems

of interconnections is presented in Figure 1. As shown here, customised resource allocators for specified multi-accelerator systems and certain types of resources have been developed in the literature. Two problems remain: (1) Most of them are based on the FIFO [2]-[4] method without heuristics which lead to the possibility of improving performance by reducing FR: in general, a sophisticated heuristic method can process more RArs. (2) They are customised for each resource and system which lead to the need for a uniform allocator that can apply on heterogeneous resources and systems without redesign. Our work provides a generalised procedure for developing a heuristic-based uniform allocator that can handle resources with arbitrary capacity and allocation request representations. While the main task to achieve uniformity and efficiency is to construct uniform heuristics for heterogeneous multitype RArs to guide the heuristic-based allocation strategy, they should be universally consistent, so they can be used to compare heterogeneous types of RArs even if each RAr can be separated into multiple accelerators. They should also be easilycomputable, within polynomial-time, so it will not increase the time complexity to guarantee the process efficiency. We first define the universal relationship between heterogeneous RArs in order to model the general RArs and rank them appropriately. 
Definition 1: Given the relationship $\leq$, named less than, between RArs on a multi-accelerator system $\alpha: \forall A, B \in$ RArs, $\alpha(B) \mapsto \alpha(A) \Leftrightarrow A \leq B$.

Here $\mathrm{P} \mapsto \mathrm{Q}$ means $\mathrm{Q}$ is true iff $\mathrm{P}$ is true. We say $\alpha(B)$ is true iff $\alpha$ can serve B. So the right hand side of the equation means that for all possible states of this multi-accelerator system which can serve $\mathrm{B}$, it can always serve $\mathrm{A}$. Then $\mathrm{A} \leq$ $\mathrm{B}$ represents the observation that A asks for less capacity from system $\alpha$ than $\mathrm{B}$, and $\mathrm{B}$ is more difficult to be satisfied than $\mathrm{A}$ in $\alpha$. We also give a more constrained relationship between RArs on $\alpha$, named covering, as follows:

Definition 2: $\forall A, B \in R A r s, \nexists C \in R A r s, A \leq C \leq B \Leftrightarrow$ $A \preceq B$.

It means $\alpha$ cannot provide such a capacity or resource which is between the need of A and B. Followed by defining relationships between RArs, we can build up a closed-topology for a general multi-accelerator system involving different RArs. Any possible RAr can be viewed as a one-dimensional RAr or the union of one-dimensional parallel RArs and the topology can be generated as a poset through the relationships. In fact, it will be a lattice structure: there is a common lower bound of all RArs which is a non-resource-need, which we denote as $\perp$; there is also a common upper bound of all RArs for any certain multi-accelerator system which asks for all the possible capacities simultaneously of this system.

\section{RANKING FOR Resource Allocation}

Now we can begin to design suitable rankings on RArs to show the level of difficulty-in-satisfying in general heterogeneous multi-accelerator systems. Universal consistency is satisfied if ranking function on RArs lattice topology can label each node to represent a consistent distance between that node to the bottom whilst preserving their initial order. The consistence means for any node in the lattice, the length of every path from that node to the bottom will be the same. An efficient way to construct rankings on modular lattice topologies is by considering the height function [1] which counts on the number of interim nodes from the bottom to the current node.

Definition 3: $\forall \mathrm{x}, \mathrm{y} \in$ lattice $\mathcal{L}$, a function $H$ is called height iff:(1) $H(x)=H(y)+1 \Leftrightarrow y \preceq x$; (2) $H(x)=1 \Leftrightarrow x=\perp$.

The difficulty appears when we consider the non-modular lattice structure for general multi-accelerator systems with arbitrary topology: if there is a node which has different interim nodes from itself to bottom by following different paths, then it cannot be easily ranked by the height function. Our approach to solve this problem is by applying Birkhoff's representation theorem to the initial non-modular lattice topology for RArs model of general multi-accelerator systems. This theorem gives us a way to transfer any general partial order set to a modular lattice composed by downsets of elements in the initial topology which can easily be ranked through the height function. As for implementing the representation process, we only need two steps. Firstly, generate the set of downsets for each RAr in the initial topology by traversal the initial set of orders between RArs. Secondly, generate the resulting modular lattice by ordering the downsets through inclusion relationships.

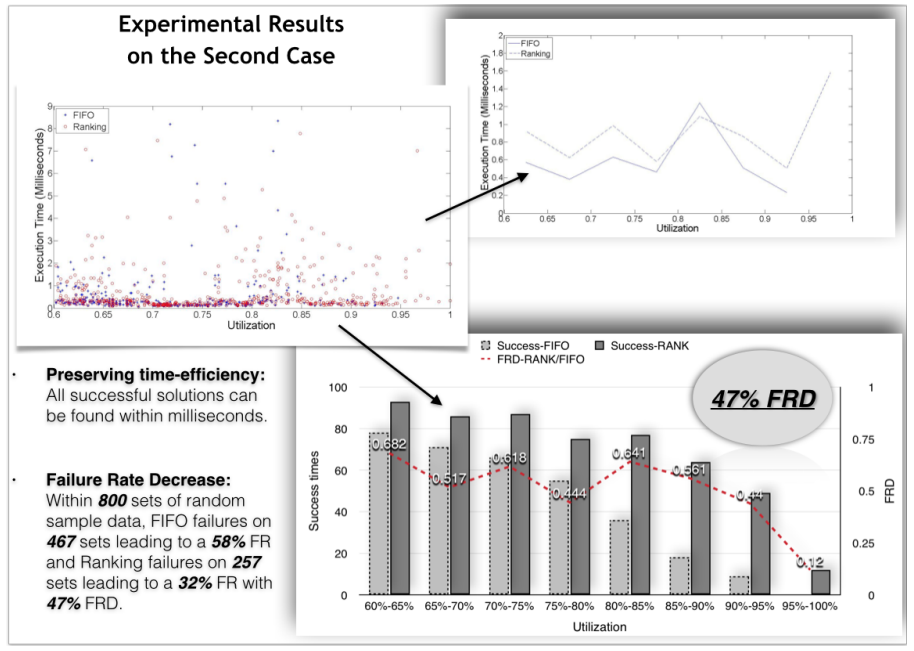

Figure 2: Results on Scalable Case

\section{EXPERIMENTAL EVALUATION}

We implement two allocation algorithms for experiments: the first one based on the FIFO method, the second one based on our ranking-based heuristic method. They are evaluated in two cases using 800 sets of random uniform distributed input data. The first case involves 3 MPC-X systems with 24 DFEs. The second case involves 15 MPC-X systems with 120 DFEs. We consider three metrics. The first is the time efficiency of the allocator, the second is the FR, which means to what percentage the method will fail to find a solution. The third is called an FRD metric to show the performance improvement of our approach against the previous approach: $F R D=\frac{\sum F R_{f}-\sum F R_{r}}{\sum F R_{f}}$. Both algorithms can finish within milliseconds to achieve time efficiency. Our ranking approach supports 33\% FRD when compared to the FIFO method in the first case, and 47\% FRD in the second case. The results for the second case are shown in Figure 2.

\section{ACKNOWLEDGEMENT}

This work is supported by Royal Academy of Engineering Research Exchanges with China and India Award 2014.

\section{REFERENCES}

[1] G. Birkhoff. Lattice theory, volume 25. American Mathematical Soc., 1940.

[2] T. Hussain, A. Haider, S. A. Gursal, and E. Ayguadé. Amc: Advanced multi-accelerator controller. Parallel Computing, 41:14-30, 2015.

[3] T. Hussain, M. Pericas, N. Navarro, and E. Ayguadé. Ppmc: Hardware scheduling and memory management support for multi accelerators. In FPL, pages 571-574. IEEE, 2012.

[4] C. Margiolas and M. F. O'Boyle. Palmos: A transparent, multi-tasking acceleration layer for parallel heterogeneous systems. In ICS, pages 307-318. ACM, 2015.

[5] Maxeler. https://www.maxeler.com, 2016.

[6] OpenStack. http://www.openstack.org, 2016.

[7] M. Stillwell, D. Schanzenbach, F. Vivien, and H. Casanova. Resource allocation algorithms for virtualized service hosting platforms. Journal of Parallel and Distributed Computing, 70(9):962-974, 2010.

[8] XtreemFS. http://www.xtreemfs.org, 2016. 